\title{
A simple Fourier transform spectrometer for terahertz applications
}

\author{
JIANG Yi, LIANG Ming, JIN BiaoBing, KANG Lin, XU WeiWei, CHEN Jian \& WU PeiHeng \\ Research Institute of Superconducting Electronics, Nanjing University, Nanjing 210093, China
}

Received August 11, 2011; accepted September 28, 2011

\begin{abstract}
A simple Fourier transform spectrometer was designed and constructed for the measurement of detectors, sources, passive devices and materials in the terahertz $(\mathrm{THz})$ range. It can be operated at frequencies between 0.3 and $1.5 \mathrm{THz}$, using a $50-\mu \mathrm{m}$-thick Mylar-film beam splitter. The spectral range can be changed by altering the thickness of the beam splitter. The highest frequency resolution is $750 \mathrm{MHz}$. We studied the properties of heterodyne detectors including superconductor mixers and semiconductor harmonic mixers, direct detectors including an InSb semiconductor bolometer, superconducting tunnel junctions and the Golay cell, and sources including Gunn oscillators and a microwave source with its multipliers, as well as various materials and passive devices including Si wafers and metal mesh filters.
\end{abstract}

Fourier transform spectrometer, direct detector, heterodyne detector, filter, terahertz

Citation: Jiang Y, Liang M, Jin B B, et al. A simple Fourier transform spectrometer for terahertz applications. Chin Sci Bull, 2012, 57: 573-578, doi: 10.1007/ s11434-011-4916-y

Because of its multiplex and etendue advantages, Fourier transform spectroscopy has become the most efficient way to evaluate the properties of signal sources, samples and detectors in the terahertz $(\mathrm{THz})$ range [1-3]. The multiplex advantage is that the interferometer receives information from the entire range of a given spectrum during each time element of a scan, whereas a grating spectrometer receives information from only a narrow band of the spectrum. The etendue advantage is that when the required frequency resolution is much lower than that determined by the throughput, the interferometer has the ability to collect a large amount of energy with no strong limitation on the resolution. This is the situation in the $\mathrm{THz}$ range. To evaluate the properties of direct detectors, heterodyne detectors (mixers) and filters fabricated in our laboratory, we designed and constructed a simple Fourier transform spectrometer (FTS) system.

\section{The FTS}

There are several types of FTS; for example, the lamellar

*Corresponding author (email: chenj63@nju.edu.cn) grating interferometer [3], Martin-Puplett interferometer [4], low-reflectivity Fabry-Perot interferometer [5] (where the low-reflectivity etalon means that the interferogram is similar to that of the Michelson interferometer) and Michelson interferometer [6]. We use a Michelson interferometer for its simplicity and compactness. It operates by separating the radiation from the source into two beams and then recombining them coherently to produce the output signal. One of the flat mirrors moves step by step during the measurement, which changes the optical path difference between the two separated beams. The signal amplitude $I$ at a given path difference $\delta$ is

$$
I(\delta)=C \int_{0}^{\infty} S(\sigma)[1+\cos (2 \pi \sigma \delta)] \mathrm{d} \sigma
$$

where $C$ is a constant, $S$ is the frequency response (spectrum) of the system, and $\sigma$ is the wave number or reciprocal of the wavelength $\lambda$. Taking the Fourier transform of both sides of eq. (1), we get

$$
S(\sigma)=C^{\prime} \int_{-\infty}^{\infty}\left[I(\delta)-\frac{1}{2} I(0)\right] \cos (2 \pi \sigma \delta) \mathrm{d} \delta,
$$


where $I(\delta)-I(0) / 2$ is the interferogram taken by the FTS, and $S(\sigma)$ is the spectrum to be measured.

$S(\sigma)$ is a product of the detector response, the relative efficiency of the beam splitter, and the spectrum of the source and spectrum of samples, as shown in Figure 1. The samples can be certain kinds of materials or passive components such as filters or metamaterials. The relative efficiency of the beam splitter can be calculated [7]. If the spectrum of the source is known, then the spectrum of the detector can be determined without a sample in the system and vice versa. By taking the reference spectrum and sample spectrum, the transmission or reflection spectrum of the sample can be determined.

\section{Construction of the FTS}

Figure 1 is a schematic diagram of the FTS showing light travelling from the source to the detector.

The broadband source consists of a hot load and a cold load. A chopper operating at frequencies between $10 \mathrm{~Hz}$ and $4 \mathrm{kHz}$ is used to switch between the two loads. The hot load is a black-body source (Shanghai Fuyuan optoelectronics HFY-206A), and its temperature can be altered between room temperature and $999^{\circ} \mathrm{C}$. The cold load is a $\mathrm{THz}$ absorber sheet (Eccosorb AN-73) immerged in liquid nitrogen, serving as a $77 \mathrm{~K}$ black body.

The incident source is collimated by the first $45^{\circ}$ off-axis parabolic mirror and then split into two parallel beams. The first parabolic mirror has a diameter of $10 \mathrm{~cm}$ and a focal length of $152.4 \mathrm{~mm}$. The diameters of the two flat mirrors are also $10 \mathrm{~cm}$. The beam splitter is made of Mylar film

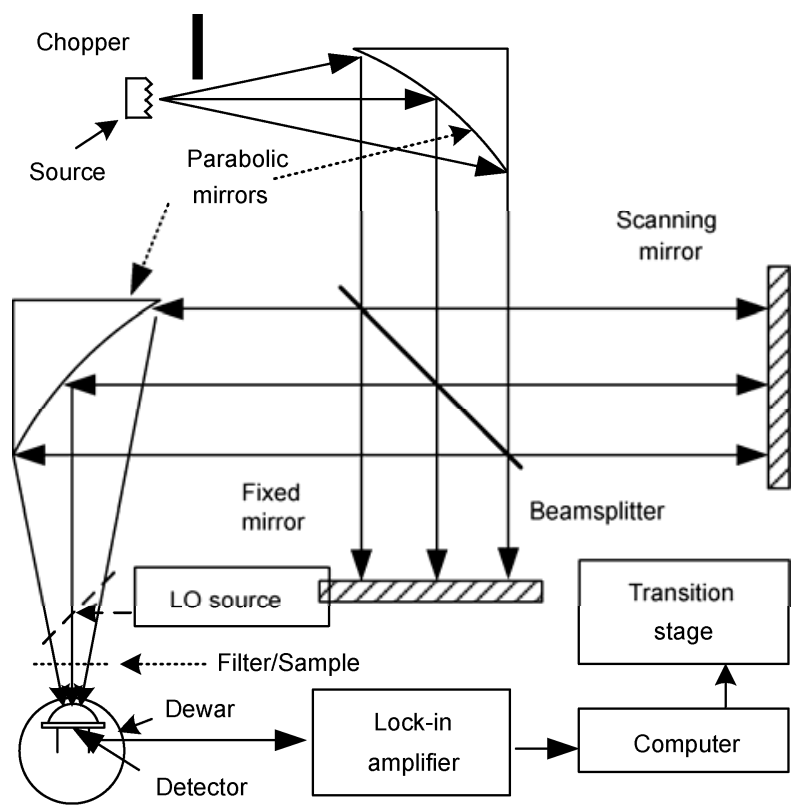

Figure 1 Schematic diagram of the FTS, which is set in a transparent plastic box that can be filled with nitrogen gas. with thickness of $50 \mu \mathrm{m}$ and could be replaced with a thicker or thinner beam splitter if necessary. Because an electromagnetic wave is reflected from both sides of the film and there is an optical path difference between the reflected beams, the transmittance and reflectance fluctuate with the frequency. The calculated beam-splitter efficiency of Mylar film with $50 \mu \mathrm{m}$ thickness is presented in Figure 2 [7,8]. After being reflected by the two flat mirrors, the two beams are recombined and focused by the second parabolic mirror, which has a focal length of $356 \mathrm{~mm}$.

The moving mirror is controlled by a high-precision electronically controlled transition stage. The minimum step distance is $2.5 \mu \mathrm{m}$. This sets the highest allowed frequency of the FTS as

$$
f_{\max }=\frac{c}{2 \times(2.5 \mu \mathrm{m} \times 2)}=30 \mathrm{THz}
$$

when a rectangular apodization function is used. The longest scanning distance of the stage is $20 \mathrm{~cm}$, which determines the highest frequency resolution of the FTS as

$$
\Delta f=\frac{c}{2 \times \text { maximum optical path difference }}=750 \mathrm{MHz},
$$

when we use a two-sided interferogram. The advantage of using a two-sided interferogram rather than a one-sided interferogram is that we can correct for the phase error caused by a displacement of the first sample from the true position having a zero optical-path difference. It also averages out asymmetries of the interferogram.

Using an extended source spreads the spectrum towards lower wave numbers [1]. The spread is $\Delta v / \nu_{0}=\Omega / 2 \pi$, the source is about $15 \mathrm{~cm}$ from the collimating mirror, and the radius of the source is $0.25 \mathrm{~cm} . \Omega \approx \pi /(60)^{2} \approx 0.00087$. Thus, $\Delta v / v_{0} \approx 0.00014$. Taking the highest value of $v_{0}$ as $1.5 \mathrm{THz}, \Delta v \approx 210 \mathrm{MHz}$, which is less than the highest frequency resolution of $750 \mathrm{MHz}$. Therefore, the resolution of the FTS system is determined by the scanning distance,

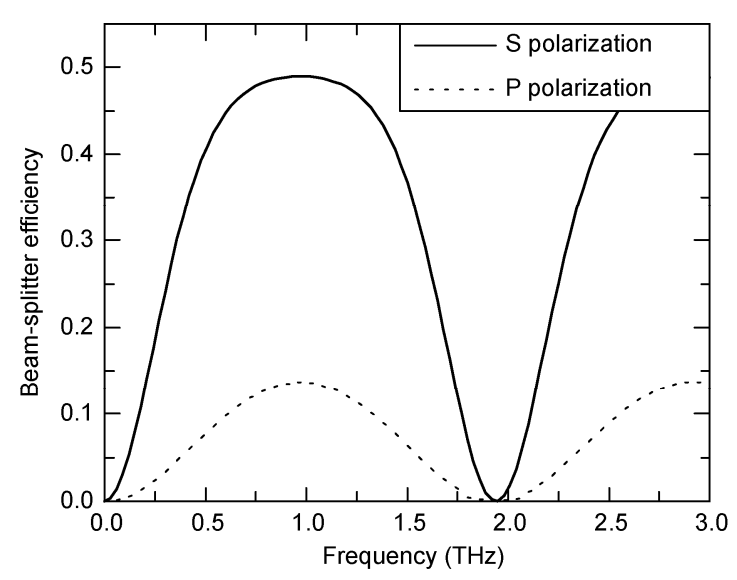

Figure 2 Calculated beam-splitter efficiencies for Mylar film with thickness of $50 \mu \mathrm{m}$ mounted at $45^{\circ}$. The refractive index is taken as 1.7 for Mylar. 
and the maximum frequency resolution is $750 \mathrm{MHz}$.

We often only use part of the transition stage and the resolution is thus less than $750 \mathrm{MHz}$, as it satisfies our needs when used in the $\mathrm{THz}$ range. The frequency range is determined by the beam splitter. When Mylar film that is 50 $\mu \mathrm{m}$ thick is used, the frequency range is approximately $0.3-1.5 \mathrm{THz}$. The range can be changed using beam splitters of other thicknesses.

A black body is used as the broadband source in our system to measure the properties of detectors and samples. The spectrum of the black body can be calculated with the Planck equation.

The whole system is constructed on an optical table with an anti-vibration structure, and is set in a plastic box. We fill the box with nitrogen gas to decrease humidity. The lowest relative humidity that can be achieved is about $2 \%$. The system is controlled by LabVIEW software installed on a personal computer. It takes about $10 \mathrm{~min}$ to complete a measurement.

The chopper frequency is set to less than the detector's modulating roll-off frequency (reciprocal of the detector's response time). The lock-in amplifier's time constant is set to about 5-10 times the chopping period. The scanning speed is limited by the integration time of the lock-in amplifier and the speed of the transition stage itself. The maximum speed is about 5 points per second. For example, for a Golay cell detector, the chopping frequency is set to $15 \mathrm{~Hz}$ and the time constant is set to $1 \mathrm{~s}$, whereas for an InSb bolometer, the chopping frequency is set to around $270 \mathrm{~Hz}$ and the time constant is set to $0.1 \mathrm{~s}$ or $0.3 \mathrm{~s}$ to achieve a higher signal-to-noise ratio (SNR).

\section{Applications of the FTS}

The useful information that we obtain from the FTS is the profile of the spectrum when measuring detectors and sources. The FTS can also measure the absorption spectrum of some materials and filters in the $\mathrm{THz}$ range.

\subsection{Direct detector measurement}

We surveyed several kinds of direct detectors; e.g. Golay cell detectors and an InSb semiconductor hot-electron bolometer (HEB) operating at $4.2 \mathrm{~K}$. These are all broadband detectors. Detectors having a narrower bandwidth, such as superconducting tunnel junctions (STJs), were also evaluated.

An InSb HEB is widely used in spectrometers, as it has a lower noise equivalent power (NEP) [9] and thus a larger SNR ratio than a Golay cell. Typical spectra of an InSb HEB at $40 \%$ relative humidity and at $10 \%$ relative humidity are shown in Figure 3. The results are normalized by the beam-splitter efficiency. The water absorption lines coincide with those reported in $[10,11]$. This verifies the correctness
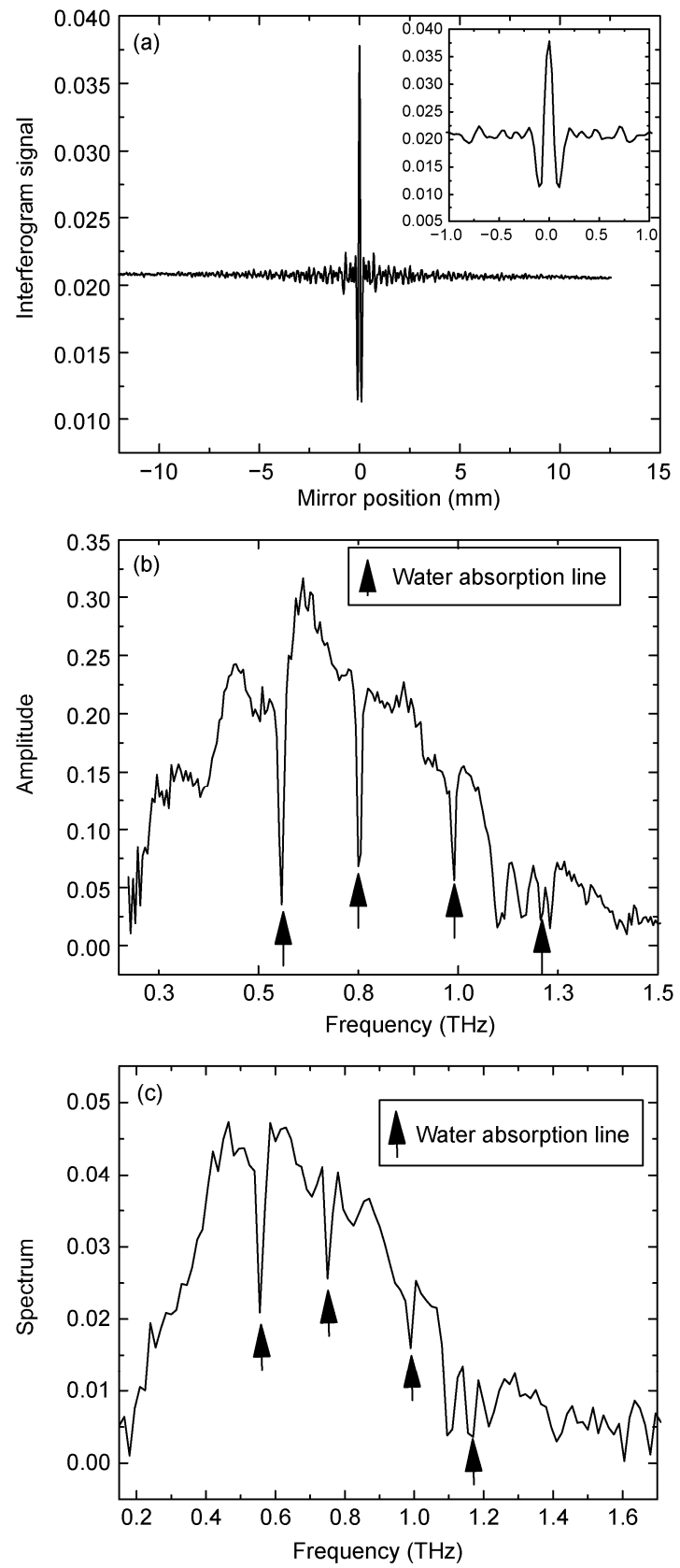

Figure 3 (a) Interferogram of an InSb HEB detector, at $40 \%$ relative humidity. (b) Spectrum of an InSb HEB detector, at $40 \%$ relative humidity; the frequency resolution is $6 \mathrm{GHz}$. The arrows show the water absorption lines. (c) Spectrum of an InSb HEB detector, at $10 \%$ relative humidity; the frequency resolution is $12 \mathrm{GHz}$. Comparing with the spectrum recorded at $40 \%$ relative humidity, this spectrum shows that water absorption is a severe problem in the $\mathrm{THz}$ range.

of our measurement. The results show that the HEB has a bandwidth of about $1 \mathrm{THz}$. Because of the wide bandwidth and relatively low NEP, bolometers operating at low temperature could be used in many applications. For example, they could be used as a detector to measure the spectrum of a source. Comparing the two results for different humidity, we see that water vapor absorption of the electromagnetic wave is quite severe in the $\mathrm{THz}$ range. 
The central frequency of the STJ direct detector was designed to be $0.65 \mathrm{THz}$ and it has a bandwidth of about 100 GHz. The NEP of the STJ detector was approximately $10^{-16}$ $\mathrm{W} / \mathrm{Hz}^{1 / 2}$ theoretically for the STJ with leakage current of about $100 \mathrm{pA}$ at $0.3 \mathrm{~K}$. The spectrum of the STJ is shown in Figure 4 and is in accordance with the designed spectrum. The SNR is not particularly high here because we use a black body as the source, and the power between the frequency ranges of 0.1 and $1.5 \mathrm{THz}$ is merely several nanowatts. STJ detectors have been reported as being used as $\mathrm{THz}$ imaging devices [12]. Compared with other types of detectors, they have the advantages of lower NEP, a greater voltage response, a faster response, a greater dynamic range and ease of use for an array with more pixels.

\subsection{Heterodyne detector (mixer) measurement}

A semiconductor Schottky diode harmonic mixer operating at room temperature and a superconductor-insulator-superconductor (SIS) mixer operating at $4.2 \mathrm{~K}$ were investigated. In our mixer system, the intermediate frequency (IF) bandwidth was limited by the IF low-noise amplifier to approximately $1 \mathrm{GHz}$ and the central frequency of the IF was 1 GHz. Resolution of about $10 \mathrm{GHz}$ was adopted in the measurement; therefore, the upper sideband and lower sideband cannot be distinguished.

The semiconductor harmonic mixer is a product of VivaTech Corporation. It operates at around $280 \mathrm{GHz}$. The measured spectrum is shown in Figure 5. The local oscillator (LO) frequency is $28 \mathrm{GHz}$; the result indicates that the mixer is a 10th harmonic mixer.

In the test of the SIS mixer, we used a $104 \mathrm{GHz}$ microwave Gunn oscillator as the LO source. The measurement result is shown in Figure 6. Harmonics in the spectrum are probably due to the LO source, which has harmonic components, and/or the mixer's nonlinear characteristics and optical misalignments. Comparing the results for the two mixers, we see that the SNR of the SIS mixer operating at $4.2 \mathrm{~K}$ is much higher than that of the semiconductor harmonic

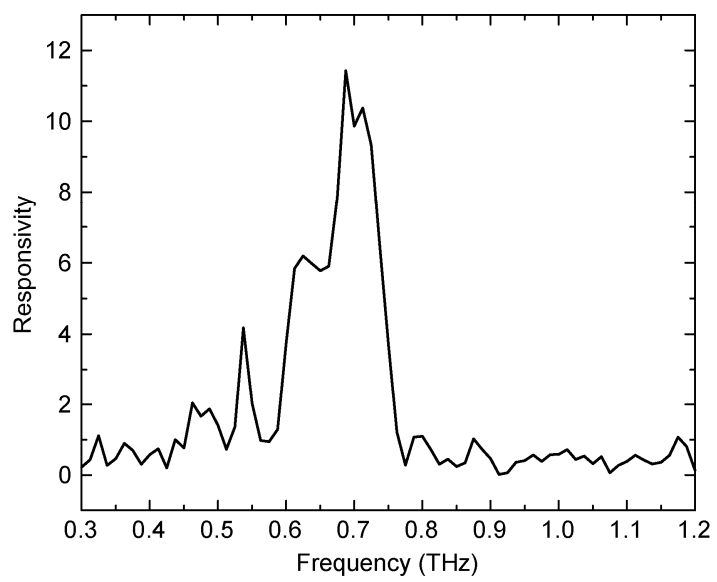

Figure 4 Spectrum of an STJ direct detector operating at a temperature of $0.4 \mathrm{~K}$.

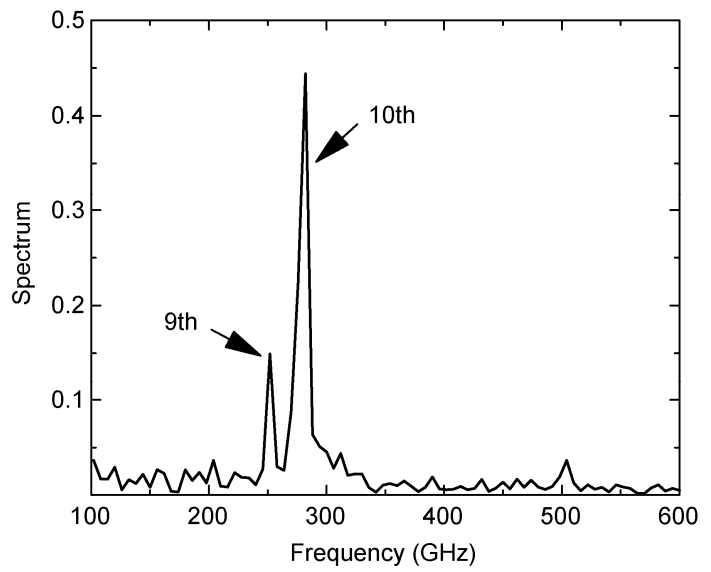

Figure 5 Spectrum of a semiconductor harmonic mixer (at room temperature).

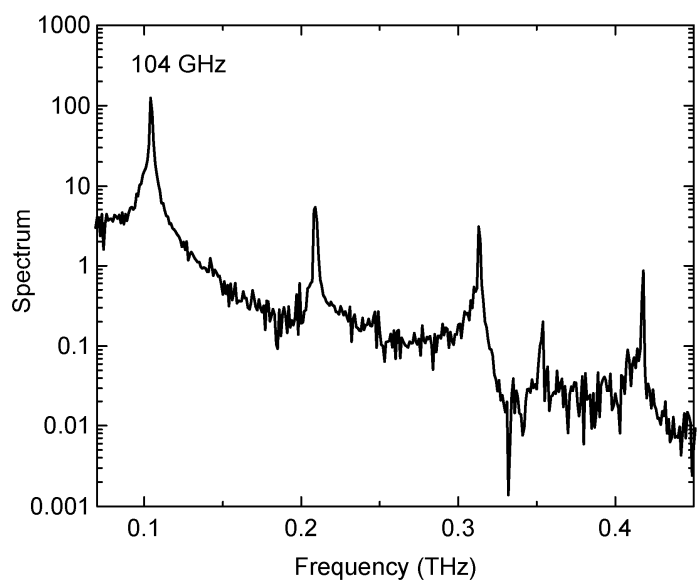

Figure 6 Spectrum of the SIS mixer $(4.2 \mathrm{~K})$. The vertical axis has a logarithmic scale.

mixer operating at room temperature. A radio-frequency bandwidth of the SIS mixer up to $8 \mathrm{GHz}$ [13] and SIS mixers made of high-temperature superconductivity materials [14] have been reported. A wide radio-frequency bandwidth means a fast measurement. If a wider bandwidth IF amplifier was used and higher resolution was employed, the RF bandwidth of the SIS mixer itself could be studied. Other types of mixers, such as superconducting HEB mixers $[15,16]$, were also tested using our system.

\subsection{Measurements of sources}

$\mathrm{THz}$ technology has developed rapidly in recent years, but one of the most difficult components to realize is the source [17]. It is important to study the characteristics of a $\mathrm{THz}$ source. Several THz sources were studied using our FTS system. They were Gunn oscillators and microwave sources with their multipliers. A Golay cell was used as the detector. The results are shown in Figure 7. The two different microwave oscillators have nearly the same output power and first harmonic frequency but differ in terms of the amplitudes 

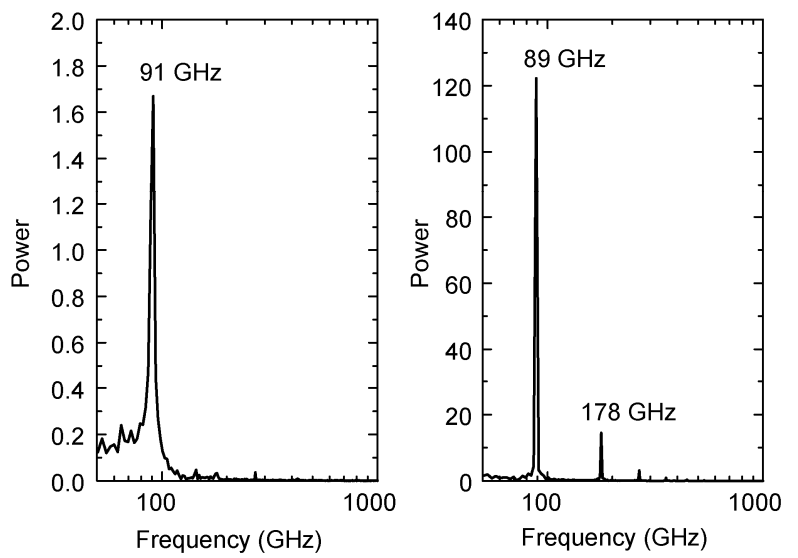

Figure 7 Spectra of two THz sources; the horizontal axis has a logarithmic scale.

of other harmonic components. Other kinds of $\mathrm{THz}$ sources such as the backward-wave oscillator, quantum cascade laser and far-infrared laser could also be tested using our FTS system.

\subsection{Measurements of filters}

It has been reported that at frequencies below $3 \mathrm{THz}$, the SNR of the FTS is worse than that of time-domain spectroscopy (TDS) [2]. The FTS system could still be used to study materials in some applications provided that there is enough SNR. Compared with the TDS, the FTS has the advantage of being easy to align while having the same frequency resolution. We measured materials that could be penetrated by $\mathrm{THz}$ waves, such as polyethylene, polytetrafluoroethylene, quartz, high-resistivity silicon ( $\mathrm{Si}$ ) [18], and metal mesh filters. Figure 8 shows a typical measured spectrum of a band-pass metal mesh filter designed to operate at a central frequency of $0.65 \mathrm{THz}$ and having a pass band of about $65 \mathrm{GHz}$. The filter is used in HEB mixer noise temperature measurements used in our laboratory [15]. The result agrees well with the designed spectrum.

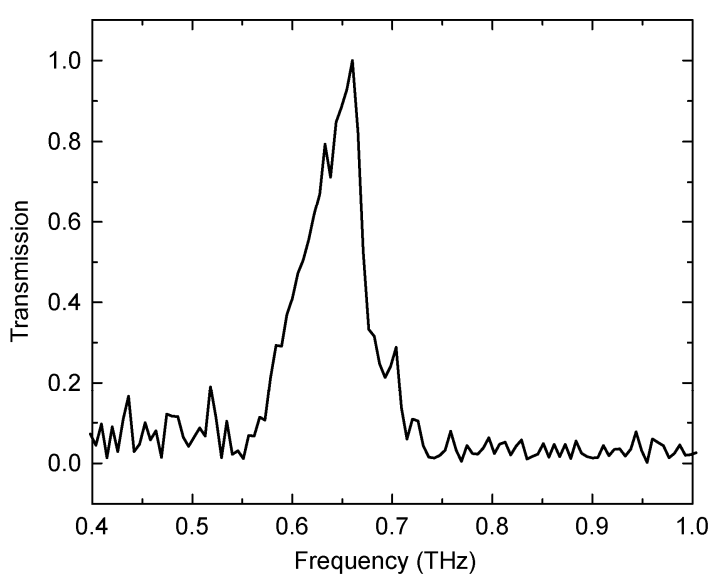

Figure 8 Spectrum of a band-pass filter.

\section{Conclusion}

We constructed an FTS system operating in the THz range, and conducted measurements of $\mathrm{THz}$ sources, detectors and passive devices. The FTS structure is simple and the optical elements are easy to align. The system works at a bandwidth of 0.3 to $1.5 \mathrm{THz}$ when using Mylar film that is 50 $\mu \mathrm{m}$ thick as a beam splitter. If a monochromatic source is tested, the 50- $\mu \mathrm{m}$-thick beam splitter can be used down to a frequency of about $50 \mathrm{GHz}$. The highest resolution of the system is $750 \mathrm{MHz}$, while we generally adopt lower resolution that is sufficient for measurements. The performance of the FTS could be improved as follows, if necessary. First, large-aperture mirrors could be introduced to advance optical throughput. Second, the length of the transition stage could be increased to improve resolution; the difficulty is the implementation and we do not need very high resolution most of the time. Third, an auto-correcting system could be used to reduce the errors caused by mirror misalignment. Fourth, a vacuum environment could be provided to eliminate the absorption effects of atmospheric water vapor.

This work was supported by the National Basic Research Program of China (2007CB310404 and 2011CBA00107), the National Natural Science Foundation of China (11173015) and the Doctoral Funds of the Ministry of Education of China (20090091110039).

1 Bell R J. Introductory Fourier Transform Spectroscopy. New York: Academic Press, 1972

2 Han P Y, Tani M, Usami M, et al. A direct comparison between terahertz time-domain spectroscopy and far-infrared Fourier transform spectroscopy. J Appl Phys, 2001, 89: 2357-2359

3 Naftaly M, Dean P, Miles R E. A simple interferometer for the analysis of terahertz sources and detectors. IEEE J Quant Electron, 2008, 14: 443-448

4 Kikuchi K, Fujii Y, Inatani J. Simple FTS measurement system for submillimeter SIS mixer. Int J IR MM Waves, 2002, 23: 1019-1027

5 Chen L J, Kao T F, Lu J Y, et al. A simple terahertz spectrometer based on a low-reflectivity Fabry-Perot interferometer using Fourier transform spectroscopy. Opt Express, 2006, 14: 3840-3846

6 Bin M, Benford D J, Gaidis M C, et al. A large throughput high resolution fourier transform spectrometer for submillimeter applications. Int J IR MM Waves, 1999, 20: 383-401

7 Weisstein E W. Millimeter/submillimeter Fourier transform spectroscopy of Jovian planet atmospheres. Dissertation for Doctoral Degree. Pasadena, California: California Institute of Technology, 1996

8 Naylor D A, Boreiko R T, Clark T A. Mylar beam-splitter efficiency in far infrared interferometers: Angle of incidence and absorption effects. Appl Opt, 1978, 17: 1055-1058

9 Putley E H. Indium antimonide submillimeter photoconductive detectors. Appl Opt, 1965, 4: 649-657

10 Pardo J R, Cernicharo J, Serabyn E. Atmospheric transmission at microwaves (ATM): An improved model for $\mathrm{mm} / \mathrm{submm}$ applications. IEEE Trans Antennas Propag, 2001, 49: 1683-1694

11 Paine S, Blundell R, Papa D C, et al. A Fourier transform spectrometer for measurements of atmospheric transmission at submillimeter wavelengths. Publ Astron Soc Pac, 2000, 112: 108-118

12 Ariyoshi S, Otani C, Dobroiu A, et al. Terahertz imaging with a direct detector based on superconducting tunnel junctions. Appl Phys Lett, 2006, 88: 203503

13 Kooi J W. Advanced receivers for submillimeter and far infrared as- 
tronomy. Dissertation for Doctoral Degree. Groningen: University of Groningen, 2008

14 Wu J B, Yi D C, Gu Z H, et al. Double-side fabrication process and millimeter wave response of intrinsic Josephson junctions. Chin Sci Bull, 2009, 54: 873-876

15 Chen J, Liang M, Kang L, et al. Low noise receivers at $1.6 \mathrm{THz}$ and $2.5 \mathrm{THz}$ based on niobium nitride hot electron bolometer mixers.
IEEE Trans Appl Supercond, 2009, 19: 278-281

16 Wang J P, Li Y B, Kang L, et al. Design and fabrication of superconducting HEB mixer. Chin Sci Bull, 2009, 54: 2013-2017

17 Siegel P H. Terahertz technology. IEEE Trans Microw Theory Tech, 2002, 50: 910-928

18 Killer D, Ediss G A, Mihaly L. Infrared measurements of possible IR filter materials. Int J IR MM Waves, 2006, 27: 835-846

Open Access This article is distributed under the terms of the Creative Commons Attribution License which permits any use, distribution, and reproduction in any medium, provided the original author(s) and source are credited. 